\title{
A new measurement algorithm for PMU in power system based on all-phase Fourier transform
}

\author{
Peng Luo ${ }^{1 *}$, Hui Fan ${ }^{1}$, Suxiang Zhang ${ }^{2}$, Xiaoguang Hao ${ }^{1}$ and Xiaowei Wang ${ }^{1}$
}

\begin{abstract}
One new vector estimation approach for phasor measurement units (PMUs) within power system is put forward, which is built upon the basis of apFFT, namely all-phase fast Fourier transform. On account of the remarkable accomplishment of apFFT when subduing property of "phase invariant" and spectral leakage, the estimation approach is capable of achieving quick evaluation of PMU phase angles, and subsequently, the spectrum correction of time-shift phase discrepancy is used so as to evaluate the amplitude and frequency parameters. The calculation flow of the algorithm is given, and the hardware design of the new PMU measuring system is completed by using ARM9 microprocessor. Simulation result indicates that the method in this paper is roughly the same to an estimator without bias for noise-free conditions, and this calculation precision is also better than the existing approach.
\end{abstract}

Keywords: Phasor measurement unit (PMU), Vector estimation, All-phase fast Fourier transform (apFFT), Phase invariant, ARM9 microprocessor

\section{Introduction}

Against the background of increasing technical growth and usage of smart grid, stability and security of electric power system is of particular significance $[1,2]$. How to construct the dynamic stability monitoring and control system for whole power grid is a problem urgent to be solved. Phasor measurement unit is built on the basis of the GPS, namely the global position system, and great accuracy timing signals, which enjoys widespread applications within wide-area measurement system so as to achieve simultaneous sampling of various nodes in the system mentioned above [3]. Various kinds of phasor measurement units (PMUs) get used in areas containing on-line parameter estimation, wide-area protection, fault location, and power grid dynamic calculation [4-6], and the PMU algorithm's performance is prone to exert direct influence on the capability of this system.

Phasor measurement algorithms chiefly get created so as to evaluate the phase angle, amplitude, and frequency parameters of electric power system, mainly including

\footnotetext{
*Correspondence: 15131116222@163.com

'State Grid Hebei Electric Power Research Institute, Shijiazhuang 050021, China

Full list of author information is available at the end of the article
}

discrete Fourier transform method, zero-crossing detection method, and Kalman filtering method [7, 8]. Because of the wonderful harmonic suppression of discrete Fourier transform (DFT) for stationary signal, this method is widely applied at present. However, when the operating frequency of the system deviates from $50 \mathrm{~Hz}$, the receiving data of PMUs cannot satisfy the condition of integral period sampling, and the frequency aliasing and spectral leakage of DFT method will cause big errors in parameter estimation. In paper [9], length of the data window gets designed to function as a variable so as to promote frequency estimation accuracy, while the computational complexity gets great increase. In paper [10], sampling frequency gets regulated flexibly by system operating frequency so as to meet the restriction of integer-period sampling. Nevertheless, bay level IEDs cannot get direct command of the sampling rate of process level in typical substations, making a problem of practical applications. Paper [11] presents one vector measurement approach on the basis of recursive DFT, which reduces the estimation error of frequency significantly. But this method cannot solve the problem of spectral leakage or frequency aliasing also, and the 
amplitude of signal is assumed to be fixed, which is not suitable for dynamic condition.

In this paper, a new power system vector measuring algorithm on the basis of all-phase fast Fourier transform (apFFT) [12-14] is proposed, in which phase, frequency, and amplitude parameters of received PMU signal can be estimated synchronously, and the input data need not be sampled by strictly integrated periods.

The remaining part of this paper is designed as shown below: Section 2 will give a brief description about the relevant concepts of all-phase spectral analysis, and the evaluation performance of phase parameters for deterministic condition will come up in Section 3. In Section 4, time-shift phase difference correcting method is put forward so as to evaluate the amplitude and frequency of observed signals. Finally, simulation results and conclusion are presented respectively.

\section{Description of current analysis method}

The apFFT's data process flow is shown in Fig. 1.

From this figure, convolution window $\boldsymbol{w}_{c}=\left[w_{c}(-N+\right.$ $\left.1), \ldots, w_{c}(-1), w_{c}(0), w_{c}(1), \ldots, w_{c}(N-1)\right]^{\mathrm{T}}$ gets framed by front window $\boldsymbol{f}$ convolved with reversal back window $\boldsymbol{b}$, which is the result of the cross-correlation operation of two window sequences with length $N$, counted by:

$$
w_{c}(n)= \begin{cases}f(n) * b(-n), & n \in[-N+1, N-1] \\ 0, & |n| \geq N\end{cases}
$$

Here, the front window $\boldsymbol{f}$ and the back window $\boldsymbol{b}$ are usually required to be the same symmetrical window, and none of them are rectangular windows. Due to the symmetry of $\boldsymbol{f}$ and $\boldsymbol{b}$, the convolution window $\boldsymbol{w}_{c}$ is also a symmetrical window as $w_{c}(n)=w_{c}(-n)$.

The FT of $\boldsymbol{w}_{c}$ is as follows:

$$
W_{c}\left(e^{j \omega}\right)=F\left(e^{j \omega}\right) B\left(e^{-j \omega}\right)=F\left(e^{j \omega}\right) B^{*}\left(e^{j \omega}\right)
$$

It is found that the Fourier transform of the whole $N$ types of data units containing point $x(0)$ are taken into full consideration in the apFFT result, which brings many excellent performance such as flat phase distribution in the vicinity of the spectral peak and suppression of amplitude spectral leakage. Besides, the FFTs of various data units are taken into account, and the calculation of apFFT is realized by simply one FFT, significantly improving the computational efficiency.

For the applications of phasor measurement in power system, the measured current and voltage signals often have typical stationary signal characteristics and are mostly single-frequency signals. For this type of input data, the window function used for data pre-processing is a key factor to improve the performance of the algorithm.

Take the most common cosine voltage amplitude signal as an example, the sampling point $N=64$, frequency sampling interval $\Delta \omega=2 \pi / 64 \mathrm{rad} / \mathrm{s}$, and three single frequency cosine signals have digital angular frequencies of $3.25 \Delta \omega, 3.375 \Delta \omega$, and $3.5 \Delta \omega$, respectively. The three groups of data were pre-processed with no-window, single-window, and double-window functions, then DFT

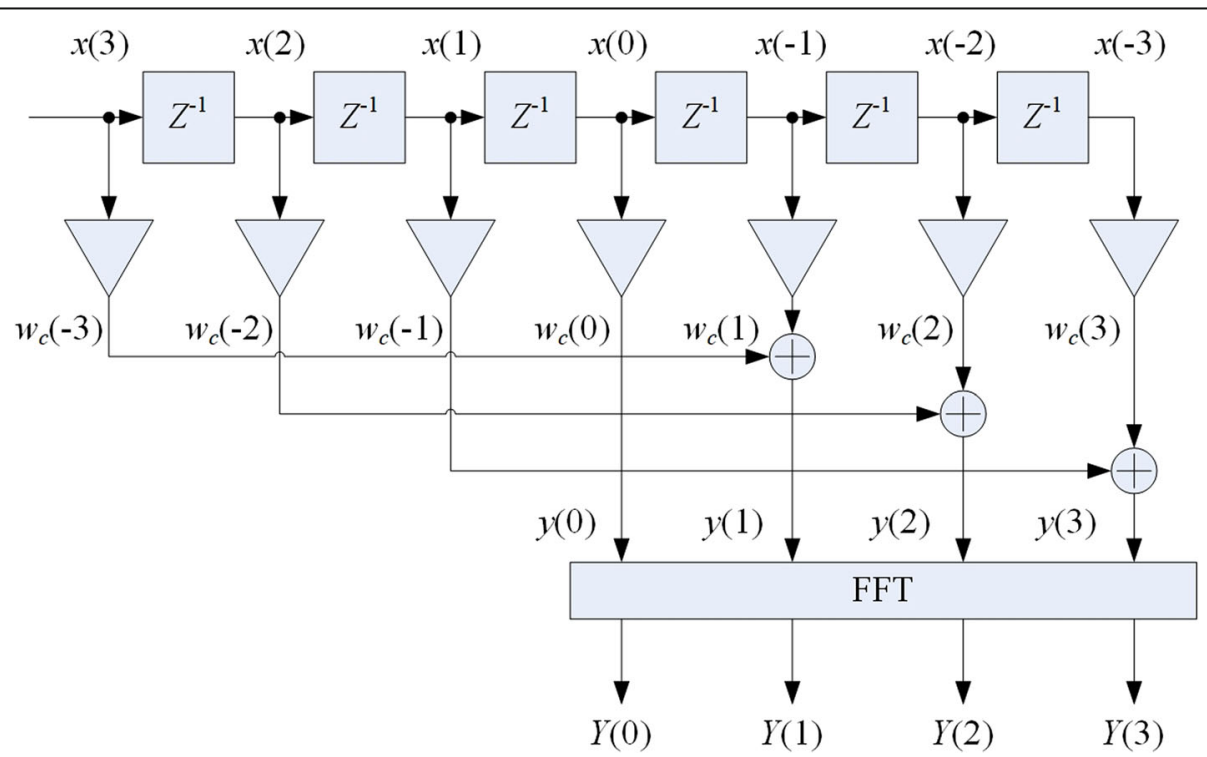

Fig. 1 apFFT spectrum analysis $(N=4)$. The data processing flow of apFFT is shown. Input sampling data are pre-treated by convolution window with length $2 N-1$, and one brand-new $N$-point sample sequence $y(n),(n=0,1, \ldots, N-1)$, is gained by the superposition of 2 data series with $N$ point interval, then the apFFT spectrum gets counted by Fourier transform of $y(n)$ 
operations were performed and the resulting apFFT discrete spectrum is shown in Fig. 2.

From the comparison results, for the single-frequency sampling data of power system, the pre-processing of window function can effectively suppress the energy leakage caused by the offset of the sampling frequency. In particular, when the frequency offset reaches $0.5 \Delta \omega$, the results of the double-window apFFT spectrum are basically only focused on the two main spectral lines. In the absence of no-window and single-window, besides the main spectral lines, there are also some side spectral leaks. Based on this situation, this paper chooses the double-window all-phase data pre-processing scheme and selects the Hanning window function with better performance against high-frequency interference.

\section{Phase estimation of deterministic signal}

Think about one compound test signal including 3 elements with different signal parameters:

$$
x(n)=\sum_{m=1}^{3} \cos \left(2 \pi \omega_{m} n / N+\phi_{m}\right), \quad n \in[-N+1, N-1]
$$

where $\omega_{1}=20.0, \omega_{2}=60.2, \omega_{3}=100.4, \phi_{1}=10^{\circ}, \phi_{2}=$ $50^{\circ}, \phi_{3}=90^{\circ}$, and $N=256$. Figures 3 and 4 have shown the windowed FFT of $x(n)$ and the double-window apFFT of $x(n)$, respectively, and rising-cosine window $\boldsymbol{w}_{h}$ is used as a window function with an expression as follows:

$$
w_{h}(n)=0.5\left[1-\cos \left(\frac{2 \pi n}{N-1}\right)\right], n \in[0, N-1]
$$

From the figures listed above, apFFT enjoys a greater feature of spectrum decrease of leakage in comparison with FFT, the leakage of energy because of non-integer process sampling, and cross talks among various frequency elements get significantly inhibited within 2 spectral lines.

When it comes to the phase spectrum, conventional FFT spectrum is irregular, and the spectral value approaches theoretical value $10^{\circ}$ at position $k=20$ (integral period sampling point), but there is a big deviation between measured phase and real phase at the points $k=$ 60 and $k=100$. The apFFT spectrum shows one organized allocation nearby object frequency, that is, the feature of "phase invariant." Using this advantage, the initial phases of all signal components can be estimated without any error correction. The phase measurement of these two approaches gets listed in Table 1.

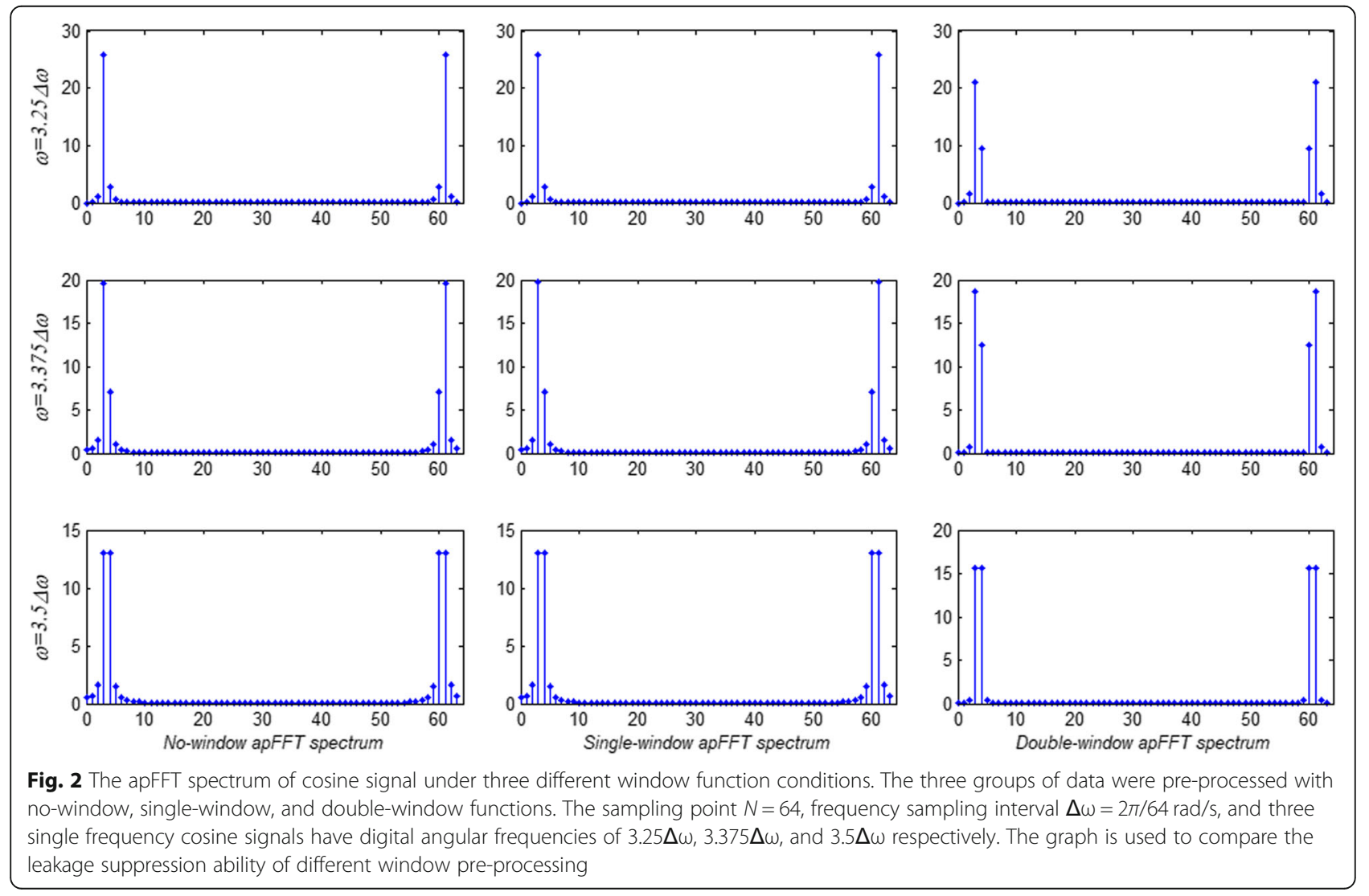



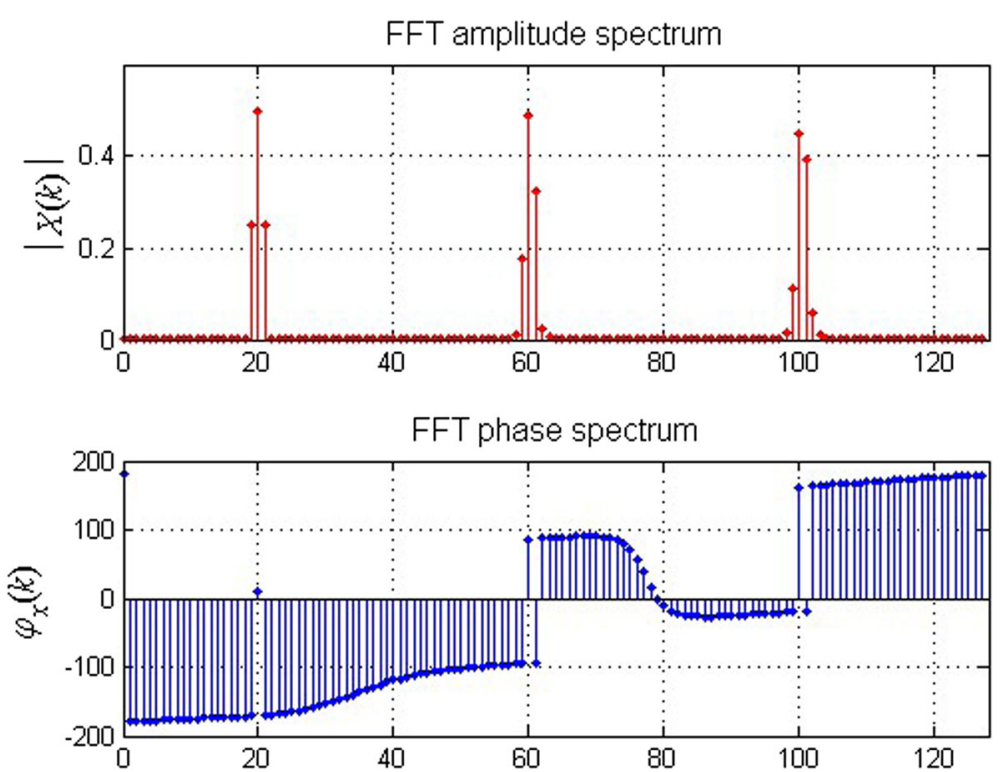

Fig. 3 FFT spectrum of $x(n)$. The FFT spectrum of the compound test signal containing three components. The upper half of the figure is the FFT amplitude spectrum of the experimental signal, and the bottom part is the FFT phase spectrum of the signal. The detailed parameters of the experimental signal were given in Section 3

\section{Frequency and amplitude estimation}

Similar to discrete Fourier transform, the barrier effect of apFFT spectral analysis also exists. If the length of calculation samples is $N$, then the minimum resolution of digital angular frequency $\Delta \omega=2 \pi / N$, and the real frequency position of signal lies between the two adjacent spectrum lines with the interval of $\Delta \omega$.
In the paper, data intercepting approach gets improvement, and brand-new time-shift phase discrepancy correcting method is put forward so as to evaluate PMU vectors' frequency and amplitude. Detailed procedures get shown below:

- Received signal is taken as a sample (perhaps not be integer process sampled) and falls into 2 sets,
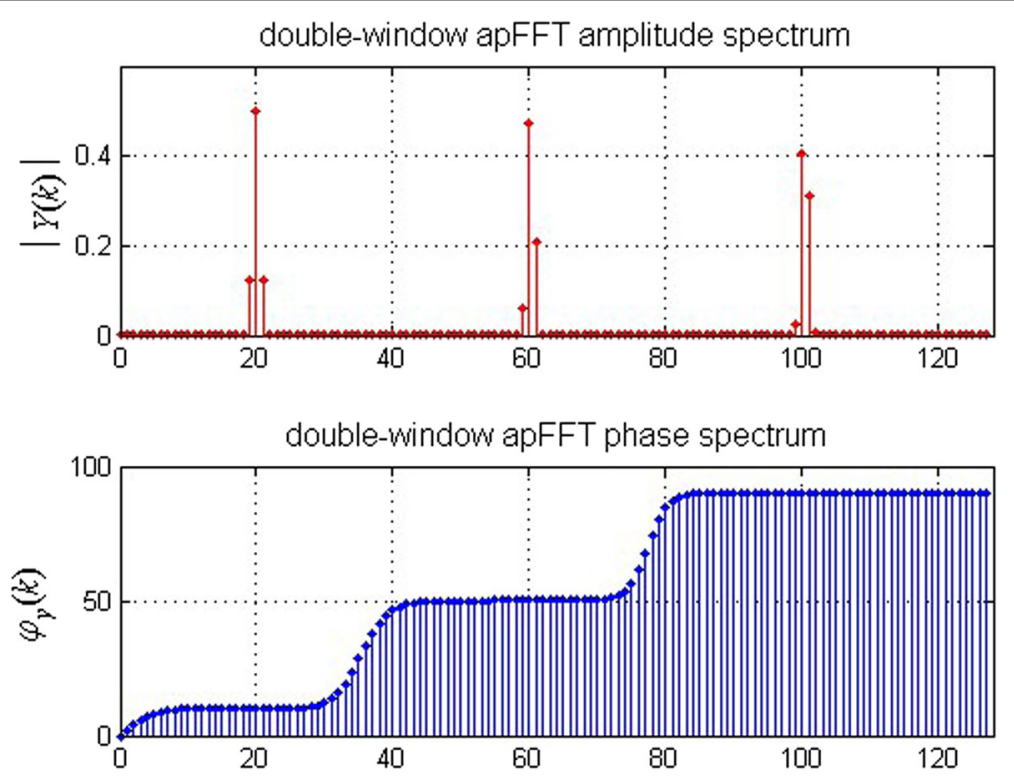

Fig. 4 Double-window apFFT spectrum of $x(n)$. The double-window apFFT spectrum of the test signal is listed in Fig. 2. The rising-cosine window is taken as window function here, and the expression of the window function is given in Eq. (4). The upper half of Fig. 3 is the apFFT amplitude spectrum of the experimental signal, and the bottom part is the apFFT phase spectrum of the signal 
Table 1 Contrast of phase measurement outcomes $(N=256)$

\begin{tabular}{llll}
\hline & Real value & Windowed FFT & Double-window apFFT \\
\hline$k=100$ & $90^{\circ}$ & $161.7187408919^{\circ}$ & $89.99999999999^{\circ}$ \\
$k=60$ & $50^{\circ}$ & $85.8592933936^{\circ}$ & $50.0000000006^{\circ}$ \\
$k=20$ & $10^{\circ}$ & $9.9997939384^{\circ}$ & $10.0000000006^{\circ}$ \\
\hline
\end{tabular}

including $x_{1}(n)$ and $x_{2}(n)$ having set length $2 N+1$.

Time delay of $x_{1}(n)$ and $x_{2}(n)$ is $n_{0}$ :

$x_{1}(n)=A e^{j\left(\omega^{*} n+\theta\right)}, n \in[-N+1, N-1]$

$x_{2}(n)=A e^{j\left[\omega^{*}\left(n-n_{0}\right)+\theta\right]}, n \in[-N+1, N-1]$

- Perform double-windowed apFFT concerning $x_{1}(n)$ and $x_{2}(n)$, and phase difference between them given by Eq. (7) is obtained from phase spectrum in the major spectral line $k^{\prime \prime}$, in which $2 n_{0} k^{\prime \prime} \pi / N$ is the compensation value of digital frequency $2 \mathrm{k}^{*} \pi / N$ of spectrum $k^{*}$ with time delay $n_{0}$ :

$\Delta \phi=\phi_{1}\left(k^{*}\right)-\phi_{2}\left(k^{*}\right)+2 n_{0} k^{*} \pi / N=\omega^{*} n_{0}$

- On the basis of Eq. (7), the evaluation of frequency with phase compensation and frequency deviation at spectral line $k^{\prime \prime}$ gets generated respectively as Eqs. (8) and (9):

$$
\begin{aligned}
& \hat{\omega}^{*}=\left[\phi_{1}\left(k^{*}\right)-\phi_{2}\left(k^{*}\right)\right] / n_{0}+2 k^{*} \pi / N \\
& d \omega=\hat{\omega}^{*}-2 k^{*} \pi / N=\left[\phi_{1}\left(k^{*}\right)-\phi_{2}\left(k^{*}\right)\right] / n_{0}
\end{aligned}
$$

- Calculate the signal amplitude by deviation of frequency $d \omega$; in the case of double-window apFFT, the evaluation equation is:

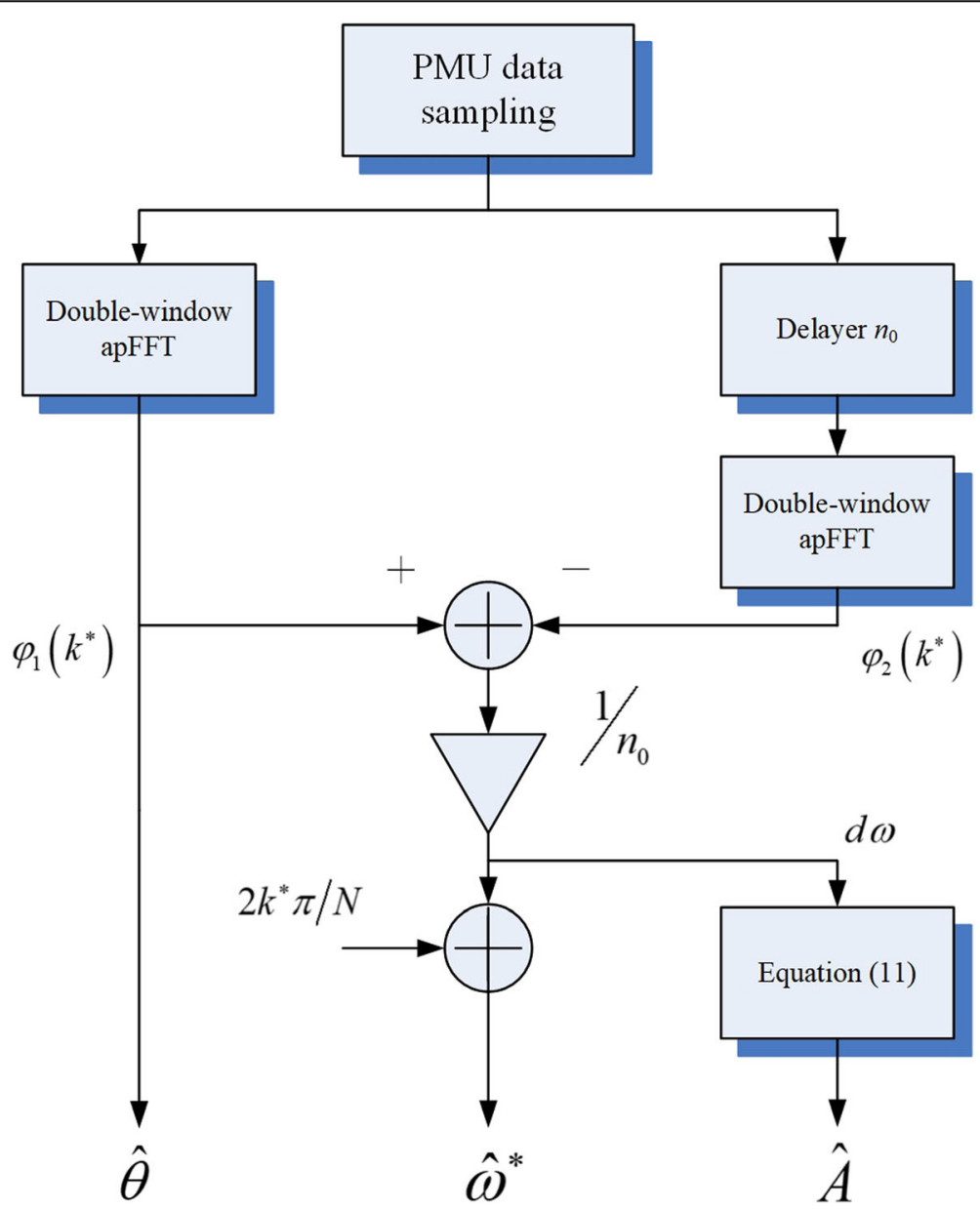

Fig. 5 Processing flow of PUM vector measurement. The processing flow of proposed PUM vector measurement algorithm on the basis of allphase spectrum analysis is listed, where the phase, frequency, and amplitude parameters of received PMU signal can be estimated synchronously, and the input data need not be sampled by strictly integrated periods 

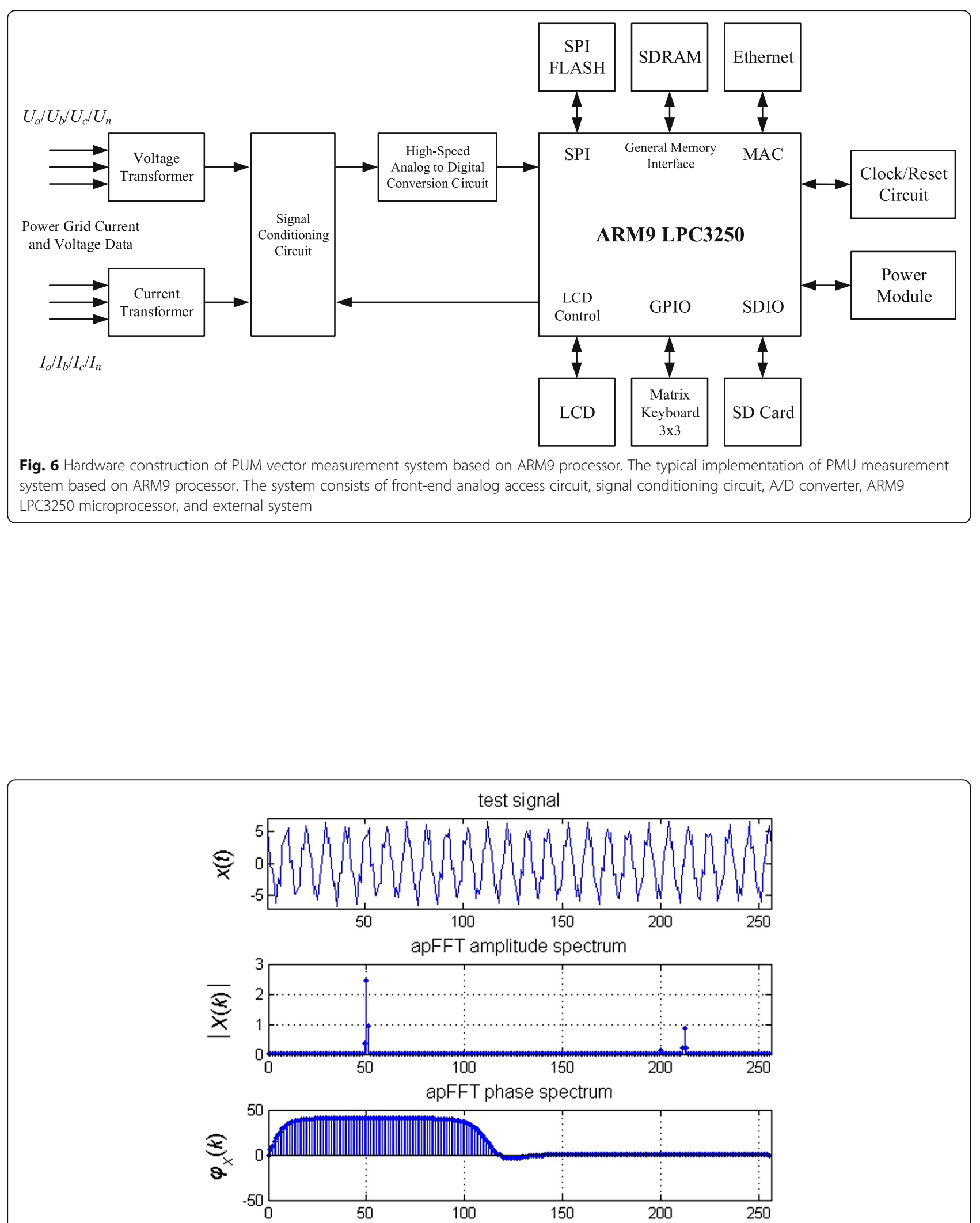

Fig. 7 Test signal $x(t)$ and the apFFT spectrum. The double-window apFFT experiment of test signal in Eq. (12) is given, which is used to confirm the performance of algorithm under harmonics condition. The first part of the figure is the time waveform of $x(t)$. The apFFT amplitude and phase spectrum are given respectively by the second and third part of the figure 
Table 2 Parameter evaluation outcomes of $x(t)$

\begin{tabular}{llll}
\hline & Real value & Approach in [9] & Approach in this paper \\
\hline$A$ & 5 & $4.9404296603^{\circ}$ & 4.9978567797 \\
$\theta$ & $40^{\circ}$ & $40.1024310871^{\circ}$ & $39.9999999996^{\circ}$ \\
$f$ & 50.5 & 50.5018709238 & 50.5000000000 \\
\hline
\end{tabular}

$\hat{A}=\frac{\left|Y\left(k^{*}\right)\right|}{F_{g}^{2}(d \omega)}$

- In the paper, the Hanning window is used for window function, then the amplitude parameter is adjusted to Eq. (11):

$$
\hat{A}=\left[\frac{2 \pi d \omega\left(1-d \omega^{2}\right) \sqrt{Y\left(k^{*}\right)}}{2 \sin (\pi d \omega)}\right]^{2}
$$

All in all, the processing flow of proposed PUM vector measuring algorithm is offered (Fig. 5).

\section{System hardware design}

In order to implement the algorithm, the optimal implementation of PMU measurement system based on ARM9 processor is shown in Fig. 6.
The system consists of front-end analog access circuit, signal conditioning circuit, A/D converter, ARM9 LPC3250 microprocessor, and external system. The front-end analog access circuit is responsible for isolating the measured $\mathrm{AC}$ signal before accessing the system and improving the security of the entire measurement system. The analog signal conditioning circuit consists of an integrated operational amplifier and a digital potentiometer, the voltage and current signals are amplified, and the digital potentiometer performs feedback resistance adjustment under the control of the ARM processor to achieve automatic variable range measurement. The ADS8568 type 8 channel synchronous sampling converter is used in the A/D conversion circuit. An industrial class ARM9 microcontroller LPC3250 is used as the control and operation core of the PMU measurement system. The external SPI-type flash memory is used to store measurement information and system parameters. The Ethernet port is used to achieve communication with the scheduling terminal. SDRAM is used to store and execute the test master program code.

\section{Simulation results and discussion 6.1 Experiment 1: Performance of algorithm under harmonics condition}

A high-precision three-phase synchronized vector measuring device is used as signal generator in this experiment. The essential frequency phase parameters of test

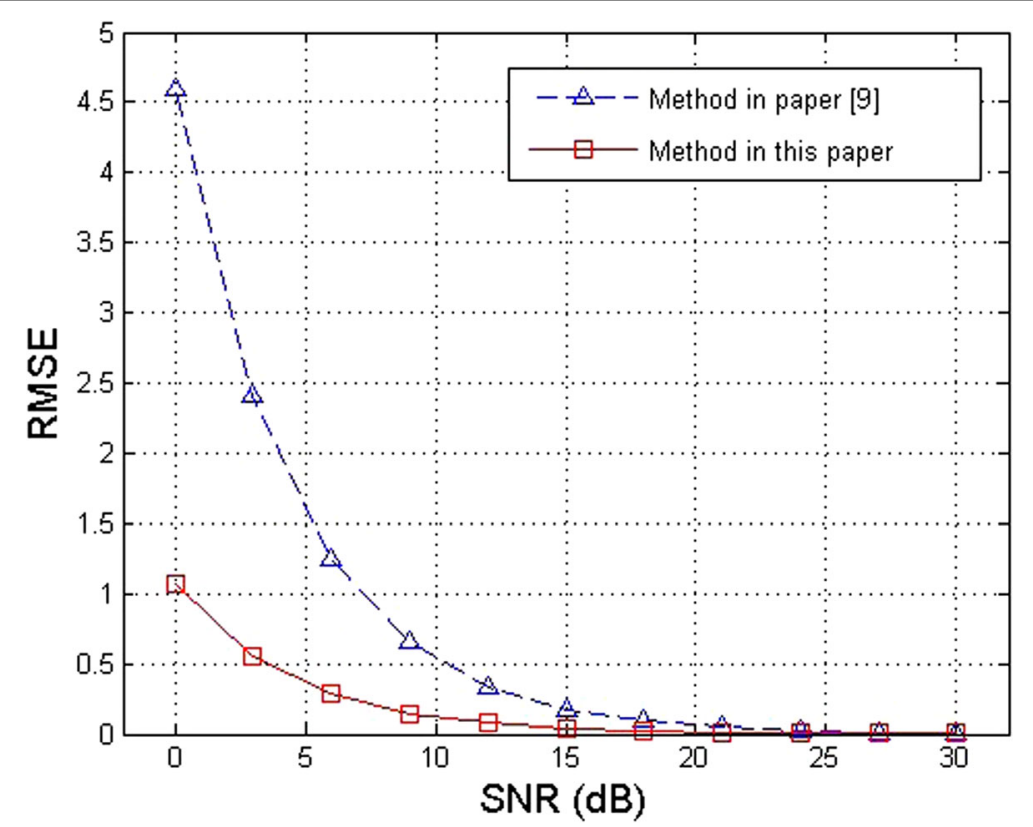

Fig. 8 Parameter estimation RMSE versus SNR. The estimation method in the paper enjoys greater performance in terms of accuracy and noise suppression in comparison with the literature method. In this contrast test, white Gaussian noise is injected into the observed signal with SNR $0 \sim 30 \mathrm{~dB}$, and the RMSE of above two parameter estimation methods is shown versus SNR 
signal get fixed with $50.5 \mathrm{~Hz}$ and $40^{\circ}$, and two harmonic signal elements with frequency $200 \mathrm{~Hz}$ and $300 \mathrm{~Hz}$ also get included in the test signal, as Eq. (12).

$$
\begin{aligned}
x(t)= & 5 \cos \left(50.5 \times 2 \pi t / f_{s}+40 \pi / 180\right)+ \\
& 0.25 \cos \left(200 \times 2 \pi t / f_{s}\right)+1.75 \cos \left(300 \times 2 \pi t / f_{s}\right)
\end{aligned}
$$

$x(t)^{\prime}$ 's double-window apFFT is given in Fig. 7.

Evaluation approaches designed in this paper and paper [9] get applied so as to evaluate the phase angle, frequency, and amplitude of this signal, and estimation results are given in Table 2.

\subsection{Experiment 2: Performance of algorithm under noise condition}

White Gaussian noise is injected into signal $x(t)$ with signal-to-noise (SNR) $0 \sim 30 \mathrm{~dB}$, and the testing flow is repeated. The root mean square error (RMSE) of above two parameter estimation methods is shown in Fig. 8 versus SNR, where the estimation RMSE of frequency, phase angle, and amplitude parameters is defined as follows:

$$
\mathrm{RMSE}=\sqrt{E\left[(\hat{f}-f)^{2}\right]+E\left[(\hat{\theta}-\theta)^{2}\right]+E\left[(\hat{A}-A)^{2}\right]}
$$

From the simulation outcomes mentioned above and comparison between the DFT and FFT method, the apFFT approach of the paper gets a greater performance in terms of accuracy and noise suppression. The algorithm of this paper can gain $7 \mathrm{~dB}$ signal-to-noise ratio gain in comparison with the traditional approach.

\section{Conclusions}

The accomplishment of PMU vector estimation algorithm tends to exert direct influence on the dependability of power system applications, like controlling, measuring, and relay protection. In the paper, one brand-new vector measuring approach for power system is put forward on the basis of all-phase spectrum analysis. In this approach, noticed data do not need to be taken as samples by thoroughly integrated processes, and the spectral leakage and barrier influence get inhibited in a significant manner. Simulation outcomes indicate that the apFFT approach enjoys a greater property in calculation accuracy and noise controlling than existing approach. There is a possibility that this approach will get widespread applications in ranges of harmonic and vector measurement explanation within power system automation, enjoying huge value of researching.

\section{Abbreviations}

apFFT: All-phase fast Fourier transform; DFT: Discrete Fourier transform; IED: Intelligent Electronic Device; PMU: Phasor measurement unit; RMSE: Root mean square error; SNR: Signal-to-noise ratio

\section{Acknowledgements \\ There are no other participants in this work except those in the author's list.}

\section{Authors' contributions}

$\mathrm{PL}$ conceived and created the research, then performed the simulations, and wrote the draft. HF and SZ reviewed and corrected the manuscript. All writers read and approved the final manuscript.

\section{Authors' information}

Not applicable.

\section{Funding}

The research was supported by the technology program of State Grid Hebei Electric Power Research Institute (No. KJ2017-016).

\section{Availability of data and materials}

Data sharing not applicable to this article as no datasets were generated or analyzed during the current study.

\section{Competing interests}

The authors declare that they have no competing interests.

\section{Author details}

${ }^{1}$ State Grid Hebei Electric Power Research Institute, Shijiazhuang 050021, China. ${ }^{2}$ State Grid Information \& Telecommunication Branch, Beijing 100053, China.

Received: 16 August 2018 Accepted: 10 June 2019

Published online: 20 June 2019

\section{References}

1. A.G. PHADKE, J.S.'. THORP, Synchronized phasor measurements and their applications (Springer, Berlin, 2008)

2. D. NOVOSEL, V. MADANI, B. BHARGAVA, et al., Dawn of the grid synchronization'. Power and Energy Magazine, IEEE 6(1), 49-60 (2008)

3. W.A.N.G. Zengping, Z.H.A.N.G. Jinfang, Q.I.A.N. Cheng, Topology error identification based on synchronized measurements for power network. Electric Power Automation Equipment 32(1), 1-8 (2012)

4. M.E.N.G. Fanchao, D.O.N.G. Xiaoliang, G.A.O. Zhiqiang, et al., Online PSS evaluation based on WAMS/PMU technology. Electric Power Automation Equipment 32(10), 146-149 (2012)

5. ZHANG Yingchen, Markham P, XIA Tao, et al. 'Wide area frequency monitoring network (FNET) architecture and applications'. IEEE Trans on Smart Grid, 2010, 1(2): 159-167.

6. D. SHI, T. D J, K.M. Koellner, et al., Transmission line parameter identification using PMU measurements. European Transactions on Electrical Power 21(4), 1574-1588 (2011)

7. P. Jafarian, M. Sanaye-Pasand, Weighted least error squares based variable window phasor estimator for distance relaying application. IEE Proceeding Generation, Transmission \& Distribution 5(3), 298-306 (2011)

8. E. GHAHREMANI, I. KAMWA, Dynamic state estimation in power system by applying the extended Kalman filter with unknown inputs to phasor measurement. IEEE Transactions on Power Systems 26(4), 2556-2566 (2011)

9. D. Hart, D. Novosel, Y. Hu, et al., A new frequency tracking and phasor estimation algorithm for generator protection. IEEE Trans on Power Delivery 12(3), 1064-1073 (1997)

10. C.H.E.N. Mingkai, Z.H.E.N.G. Xiangji, W.A.N.G. Xiaoqiang, et al., A novel algorithm for reducing spectrum leakage based on equal angle-interval sampling principle' Transactions of China Electrotechnical. Society 20(8), 9498 (2005)

11. M. AKKE, J.S. Thorp, Sample value adjustment improves phasor estimation at off-nominal frequencies. IEEE Transactions on Power Delivery 25(4), 22552263 (2010)

12. W.A.N.G. Zhaohua, H.O.U. Zhengxin, S.U. Fei, All phase FFT spectrum analysis. Journal of China Institute of Communications 24(11A), 16-19 (2003) 
13. H.U.A.N.G. Xiangdong, W.A.N.G. Zhaohua, L.U.O. Peng, et al., Discrimination and correction for dense all-phase FFT spectrums. Acta Electronica Sinica 39(1), 172-177 (2011)

14. C.H.E.N. Wei, L.I. Quan, W.A.N.G. Yangui, Object reconstruction of fouriertelescopy based on all-phase spectrum analysis. Acta Optica Sinica 30(12), 3441-3446 (2010)

\section{Publisher's Note}

Springer Nature remains neutral with regard to jurisdictional claims in published maps and institutional affiliations.

Submit your manuscript to a SpringerOpen ${ }^{\circ}$ journal and benefit from:

- Convenient online submission

- Rigorous peer review

- Open access: articles freely available online

- High visibility within the field

- Retaining the copyright to your article

Submit your next manuscript at $\boldsymbol{\wedge}$ springeropen.com 